\title{
ARTIGO ORIGINAL \\ Satisfação do paciente sobre a assistência de enfermagem hospitalar
}

Luís Guilherme Sbrolini Marques*, Letícia da Silva Schran**, João Lucas Campos de Oliveira, D.Sc. ${ }^{* *}$, Ariana Rodrigues da Silva Carvalho, D.Sc. ${ }^{* * *}$, Nelsi Salete Tonini, D.Sc. ${ }^{* * * *}$, Anair

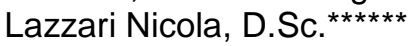

\begin{abstract}
*Especialista pela modalidade de Residência em Gerenciamento de Enfermagem em Clínica Médica e Cirúrgica pela Universidade Estadual do Oeste do Paraná (UNIOESTE), " ${ }^{*}$ PósGraduanda no Programa de Residência em Enfermagem na Especialidade de Gerenciamento de Enfermagem em Clínica Médica e Cirúrgica da UNIOESTE, ${ }^{* \star *}$ Docente colaborador dos Cursos de Graduação em Enfermagem e Residência em Gerenciamento de Enfermagem em Clínica Médica e Cirúrgica da UNIOESTE, ${ }^{* * * *}$ Professora Adjunta da UNIOESTE atuando na graduação e pós-graduação (nível Residência em Enfermagem e Mestrado Multidisciplinar co-orientação, ${ }^{* * * \star \star P r o f e s s o r a ~ a s s o c i a d a ~ a o ~ C o l e g i a d o ~ d e ~ E n f e r m a g e m ~ d a ~ U N I O E S T E, ~}$ Docente dos Cursos de Graduação em Enfermagem e Residência em Gerenciamento de Enfermagem em Clínica Médica e Cirúrgica da UNIOESTE, ${ }^{* \star * * *}$ Professora Associada, nível $A$, do Curso de Enfermagem da UNIOESTE e Coordenadora do Programa de Residência em Enfermagem na especialidade Gerenciamento de Enfermagem em Clínica Médica e Cirúrgica, Docente na área de Gerenciamento em Enfermagem. Membro do Conselho do Centro de Ciências Biológicas e da Saúde, Líder do Grupo de Pesquisa em Administração dos Serviços de Saúde e de Enfermagem
\end{abstract}

Recebido em 19 de julho de 2017; aceito em 20 de fevereiro de 2018.

Endereço de correspondência: Letícia da Silva Schran, Rua Aimorés, 562, Santo Onofre, 85806-330 Cascavel PR, E-mail: le_schran@hotmail.com; Luís Guilherme Sbrolini Marques: luisguilhermesm@hotmail.com; João Lucas Campos de Oliveira: enfjoaolcampos@yahoo.com.br; Ariana Rodrigues da Silva Carvalho: arianacarvalho@outlook.com; Nelsi Salete Tonini: nelsitonini@hotmail.com; Anair Lazzari Nicola: anairln@yahoo.com.br

\section{Resumo}

Objetivo: Avaliar a satisfação do paciente com a assistência de enfermagem hospitalar. Métodos: Pesquisa avaliativa de abordagem quantitativa. Foi desenvolvida em uma unidade de clínica médica e cirúrgica de um hospital universitário público do interior do Paraná, Brasil. Sobre uma amostra $(n=59)$ probabilística elegível de pacientes, empregou-se o Instrumento de Satisfação do Paciente (ISP), testado por Alfa de Cronbach, além da coleta de variáveis sociodemográficas e clínicas entre setembro e novembro de 2016. Os dados foram tabulados e analisados por estatística descritiva. Resultados: A amostra era predominante do sexo masculino $(54,24 \%)$ e internada pela clínica cirúrgica $(47,46 \%)$. A pontuação geral do ISP foi 3,98. Os domínios Profissional, Educacional e Confiança do ISP obtiveram avaliações em 4,14; 3,81; e 3,98, respectivamente. Conclusão: Concluiu-se que os pacientes estavam satisfeitos com o serviço de enfermagem, e que o aspecto educativo do trabalho do enfermeiro pode ser repensado.

Palavras-chave: satisfação do paciente, qualidade da assistência à saúde, enfermagem, gestão da qualidade, avaliação em saúde.

\footnotetext{
Abstract

Patient satisfaction about hospital nursing assistance

Objective: To evaluate patient satisfaction with hospital nursing care. Methods: Evaluation research of quantitative approach. This study was developed in a medical and surgical clinic unit of a public university hospital of the interior of Paraná, Brazil. For an eligible sample $(n=$ 59), the Patient Satisfaction Instrument (ISP), tested by Cronbach's Alpha, was used, as well as the collection of socio-demographic and clinical variables between September and November 2016. Data were collected tabulated and analyzed by descriptive statistics. Results: The sample was predominantly male $(54.24 \%)$ and hospitalized in the surgical clinic $(47.46 \%)$. The overall ISP score was 3.98. The ISP's Professional, Educational and Confidence domains obtained
} 
evaluations at $4.14 ; 3.81$; and 3.98 , respectively. Conclusion: It was concluded that the patients were satisfied with the nursing service, and that the educational aspect of the nurse's work can be rethought.

Key-words: patient satisfaction, quality of health care, nursing, quality management, health evaluation.

\section{Resumen}

\section{Satisfacción del paciente sobre la asistencia de enfermería hospitalaria}

Objetivo: Evaluar la satisfacción del paciente con la asistencia de enfermería hospitalaria. Métodos: Investigación de evaluación de enfoque cuantitativo. Fue desarrollada en una unidad de clínica médica y quirúrgica de un hospital universitario público del interior de Paraná, Brasil. En una muestra $(n=59)$ probabilística elegible de pacientes, se empleó el Instrumento de Satisfacción del Paciente (ISP), probado por Alfa de Cronbach, además de la recolección de variables sociodemográficas y clínicas entre septiembre y noviembre de 2016. Los datos fueron tabulados y analizados por estadística descriptiva. Resultados: La muestra era predominante del sexo masculino $(54,24 \%)$ e internada por la clínica quirúrgica $(47,46 \%)$. La puntuación general del ISP fue de 3,98. Los dominios Profesional, Educativo y Confianza del ISP obtuvieron evaluaciones en 4,14; 3,81; y 3,98, respectivamente. Conclusión: Se concluyó que los pacientes estaban satisfechos con el servicio de enfermería, y que el aspecto educativo del trabajo del enfermero puede ser repensado.

Palabras-clave: satisfacción del paciente, calidad de la asistencia sanitaria, enfermería, gestión de la calidad, evaluación en salud.

\section{Introdução}

Devido às mudanças sociais, econômicas e epidemiológicas constantes, o setor saúde precisa alavancar suas práticas de gestão rumo à melhoria contínua da qualidade dos serviços. Ainda que seja um desafio definir qualidade no âmbito da saúde por causa das peculiaridades deste setor de produção, tem-se aceito que alguns aspectos confluem para o cuidado dito qualificado, quais sejam: uso racional de recursos; diminuição do risco atrelado à assistência; e o maior grau de satisfação de usuários/clientes/pacientes, levando-se em conta os valores sociais vigentes [1].

Considerando que a melhoria da qualidade na saúde é desafiadora, reconhece-se que as práticas gerenciais em prol a este bem significam um fator de sobrevivência no mercado, além de um compromisso social proativo [2]. Dito isso, no cerne da gestão pautada na qualidade, a avaliação dos serviços se destaca como instrumento gerencial indispensável para a melhoria cíclica e sistemática da assistência [2-3].

A avaliação na saúde tem potencial para coadunar os objetivos organizacionais às melhorias no cuidado, e é necessário que as práticas avaliativas desdobrem-se sobre fatores de interesse à assistência e seus insumos [3]. Logo, a avaliação da satisfação do paciente tem sido amplamente recomendada como um dos pontos mais fortes para melhoria do cuidado, uma vez reconhecida como um dos principais indicadores de qualidade no bojo dos resultados assistenciais [1].

A satisfação do paciente é compreendida como o grau de congruência entre as suas expectativas e percepção sobre o cuidado recebido, o que reflete a avaliação cognitiva e emocional do paciente tendo como base as experiências de vida prévias [4]. No contexto da enfermagem hospitalar, que é a única categoria profissional que assiste o sujeito internado nas 24 horas do dia, a satisfação do paciente pode ser apreciada pela qualidade técnica; a continuidade da assistência; a cordialidade; eficácia dos resultados, entre outros a depender da realidade organizacional e os objetivos da avaliação [4-5]. Portanto, a pesquisa de satisfação do paciente com o serviço de enfermagem emerge como ferramenta para avaliar o cuidado à luz do seu consumidor, favorecendo a tomada de decisão do enfermeiro em busca de melhorias de interesse à assistência de enfermagem [6].

Alguns fatores podem influenciar a satisfação do paciente com o cuidado de enfermagem, destacando-se aqueles que envolvem o relacionamento entre profissionais e pacientes; o apoio afetivo; as informações sobre a saúde e o cuidado; o poder de decisão do paciente sobre as condutas assistenciais; e a competência técnica dos profissionais [7]. Ademais, pacientes satisfeitos contribuem para melhorar resultados finais de ações de saúde, como a adesão a políticas preventivas e ao tratamento [8]; o que, no cerne da enfermagem que se vale do cuidado humano - atribui qualidade ao seu trabalho [6]. 
Considerando que a satisfação do paciente com o cuidado é um dos principais indicadores que tendem a apreciar com especificidade os serviços de enfermagem [5-6], postula-se que investigar sobre o fenômeno é relevante, uma vez que pode significar um meio a conhecer mais sistematicamente as reais experiências dos usuários, e, com isso, subsidiar a gerência do cuidado para a sua melhor qualidade.

Neste escopo, as pesquisas científicas sobre satisfação do paciente ganham ainda maior relevância social e profissional para a enfermagem, ao passo que podem investigar o objeto em pauta com acurácia, favorecendo a tomada de decisão mais assertiva, bem como contribuir ao construto de conhecimento que consolida o enfermeiro como gerente do cuidado. Com isso, questionou-se: Os pacientes de uma unidade de internação hospitalar estão satisfeitos com a assistência de enfermagem? Qual é seu grau de (in)satisfação? Para responder a indagação, objetivou-se avaliar a satisfação do paciente com a assistência de enfermagem hospitalar.

\section{Material e métodos}

Estudo avaliativo, transversal, descritivo, e quantitativo. A pesquisa foi realizada em uma unidade de internação em Clínica Médica e Cirúrgica Geral de um hospital universitário público do interior do Paraná, Brasil. O hospital contempla 210 leitos exclusivamente destinados ao Sistema Único de Saúde.

A unidade pesquisada possui 31 leitos e atende pacientes a partir dos 14 anos de idade, adultos e idosos de ambos os sexos, para tratamento clínico e cirúrgico. Apesar de se caracterizar como uma unidade de internação de clínica médica e cirúrgica geral, o setor conta com dois leitos de internação preferenciais para a especialidade de cardiologia e outros dois a cirurgia bucomaxilofacial.

A população investigada foi constituída pelos pacientes internados na unidade em questão. A amostra foi estabelecida sobre a média de 145 pacientes do total de internamentos $(n=434)$ entre os meses de junho, julho e agosto de 2016. Estes dados foram obtidos por meio do software terceirizado, disponível na instituição como prontuário eletrônico do paciente.

A amostra $(n=59)$ de pacientes foi estimada através de cálculo probabilístico, com nível de confiança de $90 \%$ e margem de erro de $10 \%$. Os critérios de inclusão foram: pacientes de ambos os sexos, alfabetizados, maiores de 18 anos, e hospitalizados pelo menos 48 horas na instituição, coadunando à pesquisa em satisfação do paciente [6].

Empregou-se procedimento de adequabilidade da amostra, calculada por meio do critério de Kaiser-Meyer-Olkin (KMO), sendo o conjunto de indicadores considerados razoáveis para a análise descritiva proposta, mas não a fatorial, que não foi almejada [9], uma vez que o valor de $\mathrm{KMO}$ foi igual a 0,462. Ademais, testou-se a confiabilidade do construto do instrumento de coleta de dados pelo coeficiente Alfa de Cronbach, tendo como parâmetro satisfatório valores iguais ou acima de 0,70 [9], demonstrando que houve consistência interna entre as questões respondidas ao instrumento, ao obter-se valor de 0,89 .

A coleta de dados ocorreu no período de setembro a novembro de 2016. Para isso, foi utilizado um instrumento estruturado preenchido pelo próprio participante, e assistido pelo pesquisador quando solicitado. Na primeira etapa foi realizada a coleta de dados sociodemográficos e clínicos dos pacientes utilizando-se de um formulário próprio, constituído de: sexo, idade, estado civil, nível de escolaridade, dias de internação, e especialidade médica na internação. Em sequência, os pacientes responderam ao questionário denominado "Instrumento de Satisfação do Paciente" (ISP), que foi adaptado e validado no Brasil do instrumento norte americano original denominado Patient Satisfaction Instrument [10].

O ISP é constituído de 25 itens agrupados em três domínios: Profissional, Educacional e Confiança [10]. Os domínios Profissional e Educacional contêm sete itens cada um, que abordam, respectivamente, questões técnicas relacionadas ao cuidado e que tratam de situações referentes às atitudes educativas da equipe de enfermagem ao paciente. Por sua vez, o domínio Confiança contempla onze situações sobre relacionamento interpessoal entre profissionais e pacientes $[5,10]$.

As respostas dos itens do ISP são representadas em uma escala de medida do tipo Likert, com cinco alternativas de respostas, variando de discordo totalmente (1) a concordo totalmente (5), quanto maior a pontuação, maior o nível de satisfação do paciente com os cuidados de enfermagem [10]. Desta forma, o nível de pontuação "3" representa a neutralidade de resposta, e foi utilizada como o ponto de corte para apreciação positiva ou negativa da satisfação do paciente. 
Os itens do ISP comumente apresentam o termo "enfermeiro", mas este foi entendido e orientado aos pacientes como extensão à equipe de enfermagem hospitalar, uma vez que o instrumento foi validado do original referente ao contexto norte-americano [10], ou seja, que tem uma divisão hierárquica na equipe de enfermagem diferente da vivenciada no Brasil.

Os dados coletados à caracterização da amostra e do ISP foram compilados em planilhas eletrônicas do software do Microsoft Office Excel, versão 2010. Conforme recomendação, na tabulação dos dados, os itens de cunho negativo do ISP foram invertidos a fim de respeitar a ordem da pontuação do instrumento [10].

Aos dados tabulados procedeu-se análise estatística descritiva, com uso de medidas de proporção às variáveis de caracterização da amostra, as quais foram comparadas entre si por meio do teste de Qui-quadrado $\left(\chi^{2}\right)$ para aderência, considerando significância em $5 \%$. Aos itens e domínios do ISP, empregaram-se medidas de tendência central e dispersão. A análise dos dados foi procedida pelo programa estatístico $R \circledast$.

O nível de satisfação foi calculado por meio de: 1) Pontuação do Item: a média do item foi extraída da soma das pontuações referentes ao mesmo e dividida pelo total de pacientes; 2) Pontuação das Dimensões: a média de cada subescala/dimensão foi obtida pela soma dos pontos dos itens correspondentes a subescala, dividida pelo número total de itens que a compõem; 3) Pontuação Geral: soma das pontuações de todos os itens dividida pelo número total de itens [10].

Este estudo cumpriu com todas as exigências éticas estabelecidas na Resolução 466/2012 do Conselho Nacional de Saúde. Desta forma, o projeto de pesquisa que fomentou o estudo foi submetido e aprovado pelo Comitê de Ética em Pesquisa da Universidade Estadual do Oeste do Paraná, sob parecer de protocolo no 1560238/2016 e CAAE: 55998416.1.0000.0107.

Resultados

O estudo contou com a participação de 59 pacientes. Os pacientes do sexo masculino representaram a maior parcela da amostra, que apresentou média de idade de $43 \pm 17$ anos, e esteve internada de $5 \pm 3$ dias. Os dados de caracterização são ilustrados na Tabela I.

Tabela I - Caracterização da amostra $(n=59)$ de pacientes segundo sexo, estado civil, escolaridade e clínica de internação na unidade hospitalar. Teste Qui-quadrado para aderência. Cascavel/PR, 2016.

\begin{tabular}{|c|c|c|c|c|}
\hline Variável & Categorias & $n$ & $\%$ & p-valor \\
\hline Sexo & $\begin{array}{l}\text { Masculino } \\
\text { Feminino }\end{array}$ & $\begin{array}{l}32 \\
27\end{array}$ & $\begin{array}{l}54,24 \\
45,76\end{array}$ & 0,52 \\
\hline Estado civil & $\begin{array}{l}\text { Casado } \\
\text { Solteiro } \\
\text { Divorciado } \\
\text { Amasiado }\end{array}$ & $\begin{array}{l}27 \\
22 \\
7 \\
3\end{array}$ & $\begin{array}{l}45,76 \\
37,29 \\
11,86 \\
5,08\end{array}$ & $<0,0001$ \\
\hline Escolaridade & $\begin{array}{l}\text { Ensino Fundamental Incompleto } \\
\text { Ensino Fundamental Completo } \\
\text { Ensino Médio Incompleto } \\
\text { Ensino Médio Completo } \\
\text { Ensino Superior Completo } \\
\text { Ensino Superior Incompleto }\end{array}$ & $\begin{array}{l}16 \\
5 \\
2 \\
29 \\
4 \\
3\end{array}$ & $\begin{array}{l}27,12 \\
8,47 \\
3,39 \\
49,15 \\
6,78 \\
5,08\end{array}$ & $<0,0001$ \\
\hline Especialidade médica & $\begin{array}{l}\text { Clínica Cirúrgica } \\
\text { Clínica Médica } \\
\text { Cardiologia } \\
\text { Cirurgia Bucomaxilofacial }\end{array}$ & $\begin{array}{l}28 \\
21 \\
8 \\
2\end{array}$ & $\begin{array}{l}47,46 \\
35,59 \\
13,56 \\
3,39\end{array}$ & $<0,0001$ \\
\hline
\end{tabular}

Em relação à avaliação da satisfação do paciente propriamente dita, pelo ISP, esta obteve média geral de 3,98 pontos. A Tabela II apresenta os itens $(n=7)$ do domínio Profissional da satisfação do paciente, bem como a média geral da referida dimensão. 
Tabela II - Distribuição dos itens de avaliação da satisfação do paciente sobre o domínio Profissional. Cascavel/PR, 2016.

\begin{tabular}{lll}
\hline Domínio Profissional & Media & DP \\
\hline $\begin{array}{l}\text { O enfermeiro (a) faz questão de me mostrar como } \\
\text { seguir as orientações médicas. }\end{array}$ & 4,20 & 0,57 \\
\hline $\begin{array}{l}\text { O enfermeiro (a) está sempre muito desorganizado } \\
\text { para aparentar calma. }\end{array}$ & 3,91 & 1,01 \\
\hline O enfermeiro (a) dá bons conselhos. & 4,28 & 0,73 \\
\hline O enfermeiro (a) realmente sabe o que está falando. & 4,28 & 0,78 \\
\hline $\begin{array}{l}\text { O enfermeiro (a) é muito lento (a) para fazer as } \\
\text { coisas para mim. }\end{array}$ & 3,91 & 0,99 \\
\hline $\begin{array}{l}\text { O enfermeiro (a) não faz corretamente o seu } \\
\text { trabalho. }\end{array}$ & 4,18 & 0,91 \\
$\begin{array}{l}\text { O enfermeiro (a) é habilidoso (a) ao auxiliar o } \\
\text { médico nos procedimentos. }\end{array}$ & 4,22 & 0,63 \\
\hline Média & $\mathbf{4 , 1 4}$ & -
\end{tabular}

A Tabela III ilustra as medidas dos itens $(n=7)$ de avaliação do domínio Educacional do ISP e a média geral do domínio.

Tabela III - Distribuição dos itens de avaliação da satisfação do paciente sobre o domínio Educacional. Cascavel, PR, Brasil, 2016.

\begin{tabular}{lll}
\hline Dominio Educacional & & - \\
\hline $\begin{array}{l}\text { O enfermeiro (a) muitas vezes acha que você não é } \\
\text { capaz de entender a explicação médica sobre sua } \\
\text { doença, então ele simplesmente não se preocupa } \\
\text { em explicar. }\end{array}$ & & 1,29 \\
\hline $\begin{array}{l}\text { O enfermeiro (a) explica as coisas em uma } \\
\text { linguagem simples. }\end{array}$ & 4,30 & 0,49 \\
\hline $\begin{array}{l}\text { O enfermeiro (a) faz muitas perguntas, mas quando } \\
\text { ele (a) recebe a resposta, parece não fazer nada a } \\
\text { respeito. }\end{array}$ & 3,59 & 1,15 \\
\hline $\begin{array}{l}\text { Eu gostaria que o enfermeiro (a) me desse mais } \\
\text { informações sobre os resultados do meu exame. }\end{array}$ & 2,89 & 1,27 \\
\hline $\begin{array}{l}\text { E sempre fácil entender o que o enfermeiro (a) está } \\
\text { dizendo. }\end{array}$ & 4,25 & 0,79 \\
\hline $\begin{array}{l}\text { O enfermeiro (a) fornece as orientações na } \\
\text { velocidade correta. }\end{array}$ & 3,98 & 0,81 \\
\hline $\begin{array}{l}\text { O enfermeiro (a) sempre dá informações completas e } \\
\text { suficientes do por que os exames foram solicitados. }\end{array}$ & 3,96 & 0,97 \\
\hline \begin{tabular}{l} 
Média \\
\hline
\end{tabular} & 3,81 & - \\
\hline
\end{tabular}

Por fim, a Tabela IV sumariza os resultados de avaliação dos itens $(n=11)$ do domínio Confiança da satisfação do paciente, bem como a média geral desta dimensão.

Tabela IV - Distribuição dos itens de avaliação da satisfação do paciente sobre o domínio Confiança. Cascavel/PR, 2016.

\begin{tabular}{lll}
\hline Domínio Confiança & 3,98 & - \\
\hline $\begin{array}{l}\text { O enfermeiro (a) deveria ser mais atencioso do que } \\
\text { ele ou ela é. }\end{array}$ & 3,66 & 1,24 \\
\hline $\begin{array}{l}\text { O enfermeiro (a) é uma pessoa agradável de ter por } \\
\text { perto. }\end{array}$ & 4,55 & 0,52 \\
\hline $\begin{array}{l}\text { A gente se sente à vontade para fazer perguntas ao } \\
\text { enfermeiro (a). }\end{array}$ & 4,25 & 0,77 \\
$\begin{array}{l}\text { O enfermeiro (a) deveria ser mais amigável do que } \\
\text { ele ou ela é. }\end{array}$ & 3,13 & 1,40 \\
\hline $\begin{array}{l}\text { O enfermeiro (a) é uma pessoa que consegue } \\
\text { entender como eu me sinto. }\end{array}$ & 4,15 & 0,87 \\
\hline
\end{tabular}




\begin{tabular}{|c|c|c|}
\hline $\begin{array}{l}\text { Quando eu preciso conversar com alguém, eu posso } \\
\text { contar meus problemas ao enfermeiro (a). }\end{array}$ & 4,01 & 0,92 \\
\hline $\begin{array}{l}\text { O enfermeiro (a) está muito ocupado (a) no posto } \\
\text { para perder tempo conversando comigo. }\end{array}$ & 3,49 & 1,18 \\
\hline $\begin{array}{l}\text { O enfermeiro (a) é compreensivo (a) ao ouvir os } \\
\text { problemas do paciente. }\end{array}$ & 4,18 & 0,56 \\
\hline O enfermeiro (a) não tem paciência suficiente. & 4,06 & 1,05 \\
\hline $\begin{array}{l}\text { Estou cansado (a) do (a) enfermeiro (a) falar comigo } \\
\text { como se eu fosse uma pessoa inferior. }\end{array}$ & 4,11 & 1,07 \\
\hline $\begin{array}{l}\text { Só conversando com o enfermeiro (a) já me sinto } \\
\text { uma pessoa melhor. }\end{array}$ & 4,15 & 0,75 \\
\hline Média & 3,98 & - \\
\hline
\end{tabular}

Discussão

Sobre a caracterização da amostra estudada, mais da metade $(54,24 \%)$ dos pacientes eram homens. Na literatura, não há plena concordância sobre os resultados de pesquisa com satisfação do paciente sobre qual sexo é fator dependente de mesma, observando-se resultados em que homens se apresentaram mais satisfeitos [6], embora também haja indicação do inverso, ou seja, mulheres mais satisfeitas [5,11]. Destarte, isso reafirma que a equipe de enfermagem deve procurar o melhor atendimento ao paciente independente do seu sexo, reforçando o caráter isonômico do cuidado ético e qualificado.

Houve predomínio de pacientes com ensino médio completo (Tabela I), o que sugere que a maior parcela da amostra tinha razoável instrução escolar. Isso é um fator que merece atenção da equipe de enfermagem, uma vez que o alto nível de escolaridade pode aumentar as exigências da clientela em relação aos cuidados recebidos, mas também já foi constatado como fator que aumenta o nível de satisfação dos pacientes com a assistência de enfermagem [5].

Cumpre salientar que um dos critérios de exclusão deste estudo foi o analfabetismo, devido ter-se empregado o instrumento de forma autoaplicável, sendo necessária a interpretação do mesmo pelo participante. Todavia, observou-se dificuldade de alguns pacientes na compreensão do ISP durante sua aplicação devido às várias solicitações de auxílio, fato que pode ser repensado em pesquisas futuras, ou seja, considerar a entrevista assistida.

Em relação à idade da amostra investigada, obteve-se média de $43 \pm 17$ anos. $\mathrm{Na}$ unidade pesquisada, a maior parcela de perfil de internação cirúrgico $(47,46 \%)$ em detrimento ao clínico $(35,59 \%)$ pode ter influenciado a média de idade fora do perfil idoso, uma vez já conhecido que os pacientes atendidos pelas especialidades da clínica médica, devido ao caráter comum de doenças crônicas e sua agudização, tendem a apresentar idade superior. Neste enfoque, a literatura destaca que a idade é um fator interveniente na satisfação do paciente e que a população mais longeva tende a apresentar-se mais satisfeita [12]. Ainda assim, os pacientes inqueridos apresentaram satisfação positiva em relação aos cuidados de enfermagem recebidos.

A amostra pesquisada apresentou pontuação média geral em 3,98 pontos, considerada positiva à satisfação. As melhores avaliações sobre a satisfação do paciente se deram nos domínios Profissional $(4,14)$ e Confiança $(3,98)$, sendo o domínio Educacional o pior avaliado $(3,81)$ pelos participantes. Estes achados corroboraram pesquisas anteriores, na qual a média geral de satisfação do paciente, constatada em 3,9 no interior de São Paulo [5]; 3,7 em Minas Gerais [13] assemelharam-se à constatada neste estudo, bem como também ratificam a pior avaliação sobre o domínio Educacional do ISP.

Considera-se que o achado sobre a satisfação do paciente ao caráter educativo da equipe de enfermagem deve contribuir para se repensar o cuidado prestado, reavaliando as ações relacionadas à educação em saúde, em especial pelo enfermeiro, que precisa liderar 0 trabalho da enfermagem. Isso porque cabe ao enfermeiro fortalecer os atributos que vão além da sua capacidade técnica, enfatizando o aspecto educativo inerente à sua profissão [14].

Um estudo recente realizado com pacientes internados em unidades de clínica médica e cirúrgica geral, bem como neurologia e ortopedia do mesmo hospital de inquérito ao desta investigação, constatou que os pacientes legitimam o médico como profissional que mais 
fornece informações acerca da situação de saúde e tratamento correlato durante a hospitalização [15]. Diante disso e ao que se constatou na pesquisa, ainda que a média sobre a satisfação dos pacientes no domínio Educacional tenha sido discretamente positiva, fica evidente que este é um aspecto que merece atenção do enfermeiro, pois, uma vez gerente do cuidado, ele precisa militar pela qualidade do mesmo em todas as suas interfaces.

Em contraponto ao exposto, cabe ressaltar, no domínio Educacional, itens bem avaliados pelos pacientes, os quais evidenciam que o profissional da enfermagem, quando desenvolve atividades educativas durante o seu trabalho, o faz de maneira simples; e também, que seria sempre fácil entender o que o trabalhador diz. Tais itens sinalizam que ainda que os pacientes não possuam a melhor satisfação quanto ao aspecto educativo da equipe de enfermagem, quando a mesma o procede, o faz com clareza.

Em relação ao domínio Profissional, a maior parte dos itens de avaliação obteve pontuação média superior a quatro pontos (Tabela II). Este é um achado que denota que os pacientes legitimaram, segundo os itens de avaliação empregados, a qualidade técnica do serviço de enfermagem. Este fato merece ser considerado louvável, já que o cuidado hospitalar, ainda que complexo e permeado por diferentes facetas, é acompanhado de risco evidente à segurança do paciente internado [3], portanto que incorre na necessidade de plena habilidade técnica da equipe a fim de atenuar os riscos assistenciais e promover a melhor qualidade do cuidado.

No domínio Profissional, cumpre destacar a satisfação positiva $(4,28)$ dos pacientes sobre o item que relacionava que o enfermeiro realmente sabe o que está falando. Este item denota que os participantes acreditam no conhecimento técnico-científico dos profissionais responsáveis ao cuidado, depositando alta satisfação à capacidade técnica para a equipe de enfermagem, o que também corrobora pesquisa prévia desenvolvida no mesmo escopo em uma unidade de internação em gastroenterologia, na qual a pontuação do item foi de 4,1 [5].

Sobre a qualidade da assistência prestada pela equipe de enfermagem hospitalar e a satisfação do paciente internado, uma pesquisa concluiu que ainda que exista déficit na qualidade do cuidado avaliada in loco, a satisfação do paciente tende a ser alta/positiva [6]. Utilizando-se da literatura em pauta aos achados deste estudo, cumpre vislumbrar que mesmo que a satisfação do paciente sobre a qualidade técnica do serviço de enfermagem certamente seja um indicador plenamente válido e importantíssimo à apreciação do cuidado [4], é necessário que as organizações lancem mão de estratégias de gestão e avaliação sistêmicas da qualidade hospitalar, a fim de que a completude de informações possa contribuir mais pontual e assertivamente ao processo de melhoria.

A avaliação do paciente sobre o cuidado recebido não se baseia exclusivamente aos procedimentos técnico-assistenciais, indo além às dimensões mais subjetivas do cuidado, como aquelas que expressam confiança ou empatia por parte do profissional, domínio de interesse à mensuração da satisfação do paciente pelo ISP $[5,10]$. Nestes termos, infere-se que os relacionamentos estabelecidos no binômio equipe de enfermagem-paciente durante 0 cuidado devem priorizar as dimensões supracitadas, com a certeza de que os usuários valorizam os momentos de atenção, humanização e cuidado individualizado [13,14].

Estudo transversal realizado com 156 pacientes em unidades de internação de hospital público de Fortaleza identificou que a agilidade e a desenvoltura no atendimento é um fator relevante na satisfação do paciente com o cuidado recebido [14]. Apesar disso, a mesma pesquisa ratifica que a disponibilidade em ajudar, e a valorização do paciente como indivíduo e sua dignidade são aspectos que merecem muita atenção da equipe de enfermagem [14], portanto indo ao encontro da reflexão de que a satisfação supera o "mero" conhecimento e bom desempenho técnico da equipe cuidadora.

Em contraponto, uma pesquisa realizada com pacientes de atendimento ambulatorial, que não permaneciam internados na instituição, evidenciou que $97 \%$ dos pacientes disseram estar satisfeitos com a assistência prestada, no entanto, em menor parcela, avaliaram que carisma $(34,2 \%)$, respeito às suas decisões $(31,6 \%)$ e comunicação clara $(26,3 \%)$ seriam fatores decisivos para sua melhor satisfação [11]. Reforça-se, portanto, a peculiaridade do ambiente hospitalar, onde a fragilidade emocional, a dor e a preocupação podem influenciar na necessidade de se desenvolver empatia consolidada entre pacientes e profissionais, emergindo a importância da confiança dos clientes à sua percepção de satisfação com o cuidado.

O domínio Confiança avaliado pelo ISP aos participantes obteve a segunda melhor avaliação de satisfação entre as três dimensões de interesse do instrumento (Tabela III). Cumpre destaque a pontuação dos itens que relacionaram que o enfermeiro é uma pessoa 
agradável de ter-se por perto $(4,55)$; e que só de conversar com o enfermeiro o paciente se sentia uma pessoa melhor $(4,15)$. Postula-se que estes achados confluem à empatia e confiança do paciente perante os profissionais de enfermagem responsáveis pelo seu cuidado, uma vez demonstrada alta satisfação do paciente com a presença do profissional, bem como da sensação positiva ao remeter-se ao mesmo.

O paciente hospitalizado, sabidamente, pode experimentar situações de dor, descontentamento, medo, angústia e sofrimento. A enfermagem - que se vale da complexidade do cuidado humano - precisa intervir cuidando nas dimensões verdadeiramente humanas, promovendo a qualidade técnica de seu trabalho, como também, incorporando a dimensão subjetiva da assistência, ou seja, àquela vinculada ao conforto além do caráter físico. Dito isso, a presença do enfermeiro é fundamental na melhoria da qualidade da assistência, já que ele é o profissional responsável ao planejamento e avaliação das ações de cuidado, as quais vão além da assistência hospitalar que tende a ser focada exclusivamente nos aspectos biológicos do ser humano [15].

O enfermeiro líder, na sua relação com o paciente e equipe de enfermagem, consegue transmitir de forma consistente sentimentos de confiança e segurança que deposita na equipe que lidera, influenciando positivamente a satisfação dos pacientes, contribuindo à consolidação da qualidade organizacional [16]. Logo, a satisfação do paciente enunciada pela confiança com os profissionais de enfermagem pode ser um fator importante ao enfermeiro considerar na liderança de sua equipe, pois, utilizando-se da boa satisfação como fator promotor de melhorias no cuidado, ele poderá ter sua liderança mais bem legitimada e, assim, favorecer sua consolidação como gerente.

Conclui-se que os pacientes estavam satisfeitos com a assistência de enfermagem hospitalar. Destaca-se a melhor satisfação no domínio Profissional, seguido da dimensão Confiança. $O$ aspecto educativo do trabalho do enfermeiro, ainda que tenha sido avaliada discretamente a satisfação positiva, pode ser repensado.

As limitações mais evidentes desta pesquisa atrelam-se à amostra reduzida de pacientes e a ausência de análise de estatística inferencial, o que traz um paralelo para investigações futuras. Todavia, como a confiabilidade do construto do ISP e a adequabilidade da amostra foram testadas e aprovadas, acredita-se que o estudo contribui para o avanço da qualidade da assistência de enfermagem, uma vez que apresenta de forma clara os aspectos de interesse à melhoria do cuidado percebido pelo cliente.

Referências

1. Pena MM, Melleiro MM. Grau de satisfação de usuários de um hospital privado. Acta Paul Enferm 2012; 25(2):197-203.

2. Vituri DW, Évora YDM. Total Quality Management and hospital nursing: an integrative literature review. Ver Bras Enferm 2015; 68(5):945-52.

3. Tres DP, Oliveira JLC, Vituri DW, Alves SR, Rigo DFH, Nicola AL. Qualidade da assistência e segurança do paciente: avaliação por indicadores. Cogitare Enferm 2016; 21(5):1-8.

4. Mrayyan MT. Jordanian nurses' job satisfaction, patients' satisfaction and quality of nursing care. Int Nurs Rev 2006;53(3):224-30.

5. Dorigan GH, Guirardello EB. Satisfação do paciente em uma unidade de gastroenterologia. Acta Paul Enferm 2010;23(4):500-5.

6. Freitas JS, Silva AEBC, Minamisava R, Bezerra ALQ, Souza MRG. Qualidade dos cuidados de enfermagem e satisfação do paciente atendido em um hospital de ensino. Rev Latinoam Enferm 2014;22(3):454-20.

7. Lima CA, Santos BTP, Andrade DLB, Barbosa FA, Costa FM, Carneiro JA. Qualidade dos prontos-socorros e prontos-atendimentos: a satisfação dos usuários. Einstein 2015;13(4):587-93.

8. Bastos GAN, Fasolo LR. Fatores que influenciam a satisfação do paciente ambulatorial em uma população de baixa renda. Rev Bras Epidemiol 2013;16(1):114-24.

9. Hair JF, Anderson RE, Tatham RL, Black WC. Análise multivariada de dados. 6a . ed. Porto Alegre: Bookman; 2009. 688p. 
10. Oliveira AML. Satisfação do paciente com os cuidados de enfermagem: adaptação cultural e validação do Patient Satisfaction Instrument [Dissertação]. Campinas: Faculdade de Ciências Médicas da Universidade Estadual de Campinas; 2004.

11. Silva LC, Duprat IP, Correia MS, Ramalho HTP, Lima JA. Satisfação do paciente oncológico diante da assistência de enfermagem. Rev Rene 2015;16(6):856-62.

12. Junior JRML, Sardinha AHL, Gonçalves LHT, Coutinho NPS, Pasklan ANP, Asklan ANP, et al. Cuidados de enfermagem e satisfação de idosos hospitalizados. O Mundo da Saúde 2015;39(4):419-32.

13. Ferreira PHC, Guedes H, Moreira SAA, Baracho VS, Caldeira ABR, Guedes CF, et al. Satisfação dos clientes externos quanto aos cuidados de enfermagem. Rev Min Enferm 2016;20(1):e975. doi:10.5935/1415-2762.20160045

14. Oliveira RM, Pereira MM, Feitosa PG, Lima AS, Brito YCF, Leitão IMTA et al. Satisfação do paciente com os cuidados de enfermagem: que dimensões se sobressaem? Enferm Foco 2014;5(3/4):70-74.

15. Pedro DRC, Silva GKT, Molin TD, Oliveira JLC, Nicola AL, Tonini NS. Conhecimento do paciente sobre a assistência hospitalar recebida durante sua internação. Rev Min Enferm 2016;20(1):e978. doi: 10.5935/1415-2762.20160048

16. Nunes EMGT, Gaspar MFM. Leadership in nursing and patient satisfaction in hospital context. Rev Gaúcha Enferm 2016;37(2):e55726. doi: 10.1590/19831447.2016.02.55726 